Article

\title{
Spin Absorption Effect at Ferromagnet/Ge Schottky-Tunnel Contacts
}

\author{
Michihiro Yamada ${ }^{1}$ (D), Yuichi Fujita ${ }^{1}$, Shinya Yamada ${ }^{1,2}$, Kentarou Sawano ${ }^{3}$ \\ and Kohei Hamaya $1,2, *$ \\ 1 Graduate School of Engineering Science, Osaka University, 1-3 Machikaneyama, Toyonaka 560-8531, Japan; \\ michihiro@ee.es.osaka-u.ac.jp (M.Y.); yuichifujita141@s.ee.es.osaka-u.ac.jp (Y.F.); \\ yamada@ee.es.osaka-u.ac.jp (S.Y.) \\ 2 Center for Spintronics Research Network, Graduate School of Engineering Science, Osaka University, \\ 1-3 Machikaneyama, Toyonaka 560-8531, Japan \\ 3 Advanced Research Laboratories, Tokyo City University, 8-15-1 Todoroki, Tokyo 158-0082, Japan; \\ sawano@tcu.ac.jp \\ * Correspondence: hamaya@ee.es.osaka-u.ac.jp
}

Received: 23 December 2017; Accepted: 15 January 2018; Published: 17 January 2018

\begin{abstract}
We study the influence of the junction size in ferromagnet (FM)/semiconductor (SC) contacts on four-terminal nonlocal spin signals in SC-based lateral spin-valve (LSV) structures. When we use FM/Ge Schottky-tunnel junctions with relatively low resistance-area products, the magnitude of the nonlocal spin signal depends clearly on the junction size, indicating the presence of the spin absorption effect at the spin-injector contact. The temperature-dependent spin signal can also be affected by the spin absorption effect. For SC spintronic applications with a low parasitic resistance, we should consider the influence of the spin absorption on the spin-transport signals in SC-based device structures.
\end{abstract}

Keywords: semiconductor spintronics; germanium; spin absorption

\section{Introduction}

Spin-based electronics (spintronics) are expected for one of the beyond CMOS technologies because of the advantages of nonvolatility, reconstructibility, and low power consumption [1-5]. In the field of semiconductor-based spintronics, electrical spin injection and detection in GaAs have so far been demonstrated [6-9]. Taking the compatibility with existing Si-based electronic devices into consideration, it is important to electrically inject and detect spins in group-IV semiconductors such as Si [10-18] and Ge [19-27]. However, due to the large conductivity (spin resistance) mismatch between ferromagnet (FM) and semiconductor (SC) [28], it has been generally recognized that tunnel barriers should be inserted at the FM/SC interface for electrical spin injection and detection in SC [29-31]. Actually, many studies have utilized insulating tunnel barriers such as $\mathrm{MgO}$ for injecting spins into Si $[10-12,16,18]$ and Ge [19-21,23].

On the other hand, for the spin injection into nonmagnetic metals (NMs), the size of FM/NM junctions was frequently important because of the spin absorption effect at the FM/NM interface [32-34]. In general, the spin resistance of FM is defined as $2 \lambda \rho /\left[S\left(1-\alpha^{2}\right)\right][30,32]$, where $S$ is the effective cross-sectional area, $\alpha$ is the spin polarization, and $\lambda$ and $\rho$ are the spin diffusion length and resistivity, respectively. Owing to the unique values of $\lambda, \alpha$, and $\rho$ in FM, reducing the size of $S$ enables the increase in the spin resistance of FM, resulting in the suppression of the back flows of spins from NM towards FM [32-34]. Yang et al. demonstrated the generation of a giant pure spin current, leading to the magnetization switching of the nanomagnet by reducing the FM/NM junction size [33]. 
Even in $\mathrm{CoFe} / \mathrm{MgO} / \mathrm{n}$-Si $\left(\sim 10^{19} \mathrm{~cm}^{-3}\right)$ systems, Ishikawa et al. indicated the significance of the spin absorption effect [35]. Since relatively large junction $\left(\sim 200 \mu \mathrm{m}^{2}\right)$ sizes were used compared to the case of metallic FM/NM junctions, they took into account the value of the resistance-area product $(R A)$ of the contacts in ranging from 10 to $20 \mathrm{k} \Omega \cdot \mu \mathrm{m}^{2}$ [35]. On the other hand, Dushenko et al. recently reported the spin injection from Py into $n-G e\left(\sim 10^{19} \mathrm{~cm}^{-3}\right)$ by using a spin-pumping method through the Ohmic interface with very large contacts $\left(\sim 10^{5} \mu \mathrm{m}^{2}\right)$ [27]. This means that the spin absorption effect due to the large difference in the spin resistance between Py and Ge is ignored despite the Ohmic junction with a large contact area. From these experimental points of view, the presence of the spin absorption effect at FM/SC interfaces remains an open question.

Until now, by using reliable four-terminal nonlocal voltage measurements [36-38], we have demonstrated electrical spin injection/detection in $n$-Ge $\left(\sim 10^{19} \mathrm{~cm}^{-3}\right)$ via epitaxial $\mathrm{FM} / \mathrm{Ge}$ Schottky-tunnel junctions $[25,26,39,40]$, where the values of $R A\left(<0.5 \mathrm{k} \Omega \cdot \mu \mathrm{m}^{2}\right)$ were much smaller than those in FM/MgO/Ge junctions [19-21,23]. In this paper, to explore the spin absorption effect at the epitaxial FM/Ge Schottky-tunnel interfaces, we study the junction-size dependence of four-terminal nonlocal spin signals in lateral spin valve (LSV) devices with $R A \sim 0.4 \mathrm{k} \Omega \cdot \mu \mathrm{m}^{2}$. At low temperatures, the magnitude of spin signals evidently depends on the size of the epitaxial FM/Ge Schottky tunnel junctions. Note that the smaller junctions enable us to observe the spin signals at higher temperatures. For SC spintronic applications [41] with a low parasitic resistance, we should consider the influence of the spin absorption on the spin-transport signals in SC-based device structures.

\section{Samples and Fabrication Procedures}

Ge-based LSV structures with FM/Ge Schottky-tunnel contacts were fabricated as shown in Figure 1. By molecular beam epitaxy (MBE), we first grew an undoped Ge(111) layer ( $28 \mathrm{~nm})$ at $350^{\circ} \mathrm{C}$ (LT-Ge) on a commercial undoped $\mathrm{Si}(111)$ substrate $(\rho \sim 1000 \Omega \mathrm{cm})$. Next, an undoped $\mathrm{Ge}(111)$ layer $(\sim 70 \mathrm{~nm})$ at $700{ }^{\circ} \mathrm{C}(\mathrm{HT}-\mathrm{Ge})$ was grown on top of the LT-Ge [42]. As the spin transport layer (n-Ge), we grew a 70-nm-thick phosphorous (P)-doped $n$-Ge(111) layer (doping concentration $\sim 10^{19} \mathrm{~cm}^{-3}$ ) by $\mathrm{MBE}$ at $350^{\circ} \mathrm{C}$ on top of the HT-Ge layer. To achieve Schottky tunnel junction, the $\mathrm{P} \delta$-doping with an ultra-thin Si insertion was performed between FM and $n$-Ge channel [43]. The $\mathrm{P}+\mathrm{Si} \delta$-doped Ge layer enables us to promote the tunnel conduction and to precisely adjust the $R A$ values at $F M / G e$ junctions [44]. Here we used a $\mathrm{Co}_{2} \mathrm{FeAl}_{0.5} \mathrm{Si}_{0.5}(9 \mathrm{~nm}) / \mathrm{CoFe}(1.5 \mathrm{~nm})$ bilayer structure as the FM spin injector/detector, as previously shown in Ref. [39]. If we used highly spin-polarized FM/Ge contacts as a spin injector and detector, we may not have been able to explore the spin absorption effect because of the achievement of the high spin resistance of the FM/Ge contacts. Since $\mathrm{Co}_{2} \mathrm{FeAl}_{0.5} \mathrm{Si}_{0.5}$ was well known to be a half-metallic material [45], we intentionally inserted a conventional FM, CoFe, between $\mathrm{Co}_{2} \mathrm{FeAl}_{0.5} \mathrm{Si}_{0.5}$ and $\mathrm{Ge}$. Thus, the large enhancement in the spin resistance of the FM/Ge contacts can be ignored. The growth of the epitaxial FM layers on Ge was already shown elsewhere $[25,46-48]$. Finally, by using electron beam lithography and Ar ion milling, the grown FM/ $n$-Ge layers were patterned into the FM contacts with various sizes $(S=L \times W$, shown in Figure $2 \mathrm{~b})$, ranging from $0.8 \mu \mathrm{m}^{2}$ to $10 \mu \mathrm{m}^{2}$. The FM contacts had shape-induced anisotropy along the longitudinal direction and almost single magnetic domain in these small sizes. Contact 2 had rhomboid shape to induce the large shape anisotropy, leading to the difference in the magnetization switching field between contact 2 and contact 3. For spin transport measurements, since the external magnetic fields were applied along the longitudinal direction of the contacts, single-domain-like magnetization reversal processes can be expected. Here the $\mathrm{P} \delta$-doped layer was removed in the region of the spin-transport channel by using Ar ion milling, as shown in Figure 1.

The edge-to-edge distance between the FM/Ge Schottky-tunnel contacts was designed to be $\sim 0.4 \mu \mathrm{m}$. To fabricate Ohmic contacts as the reference electrodes, we removed the grown $\mathrm{Co}_{2} \mathrm{FeAl}_{0.5} \mathrm{Si}_{0.5} / \mathrm{CoFe}$ bilayer by using $\mathrm{Ar}$ ion milling techniques. After the removal, we formed the $\mathrm{Au} / \mathrm{Ti}$ bilayer by electron beam evaporation. 


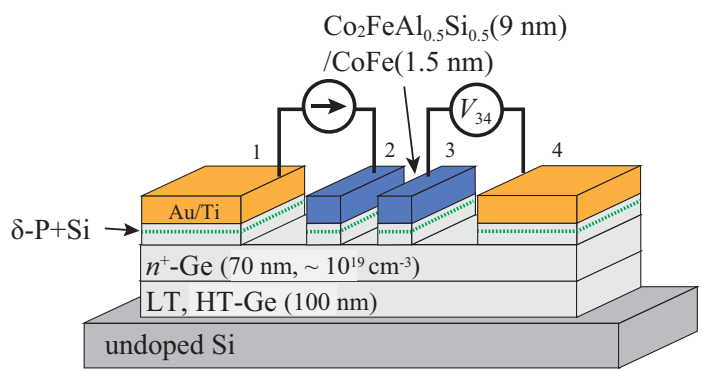

Figure 1. Schematic illustration of the fabricated lateral spin valve with ferromagnet (FM)/Ge Schottky-tunnel contacts. HT: high-temperature-grown; LT: low-temperature-grown.

(a)

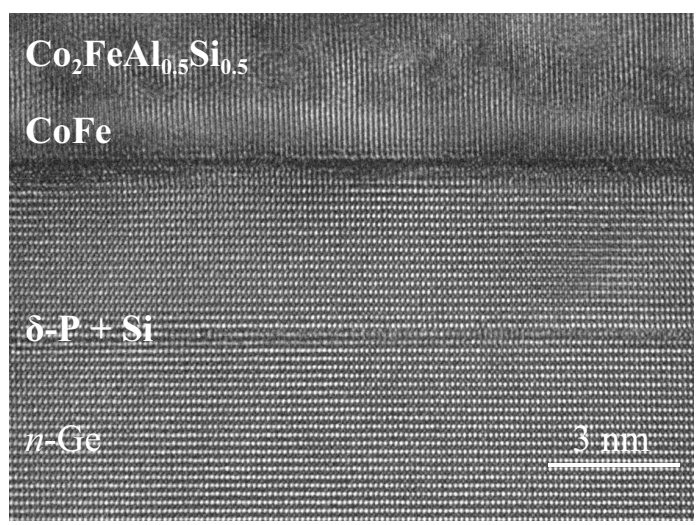

(b)
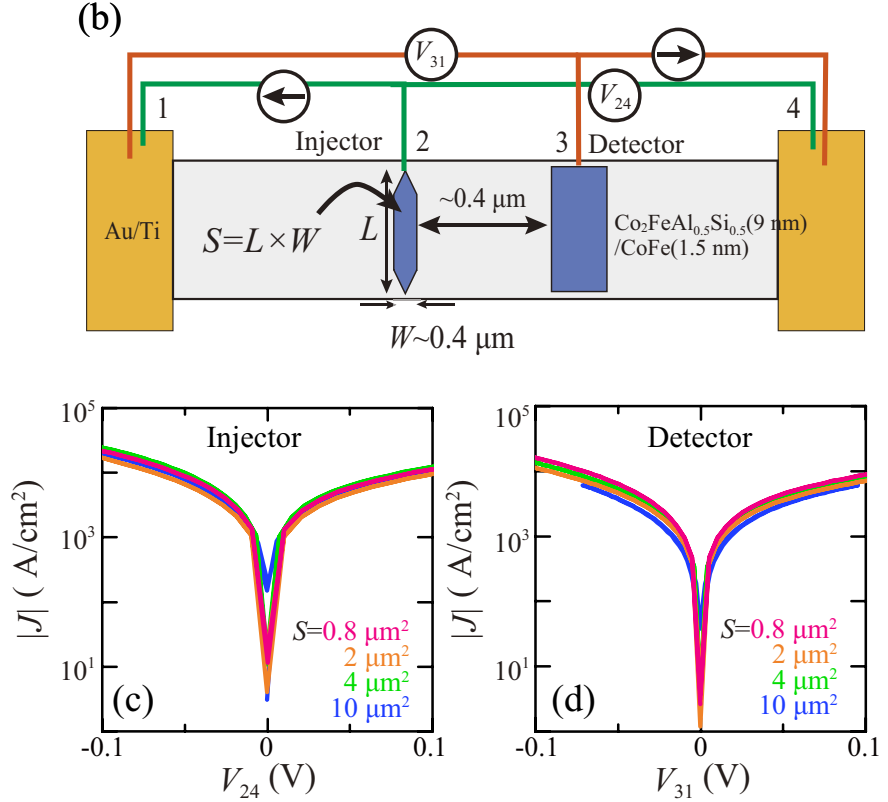

Figure 2. (a) Cross-sectional TEM image of the FM/n-Ge interface of a measured lateral spin valve (LSV) device; (b) Schematic illustration of the terminal configuration for $|J|-V$ measurements; $|J|-V$ curves for (c) spin injector and (d) spin detector with various contact sizes at $30 \mathrm{~K}$.

\section{Results and Discussion}

Figure 2a is a cross-sectional transmission electron microscope (TEM) image of a $\mathrm{Co}_{2} \mathrm{FeAl}_{0.5} \mathrm{Si}_{0.5} /$ $\mathrm{CoFe} / \mathrm{Ge}$ contact in the fabricated LSVs used for spin transport measurements. The abrupt interface 
between FM and Ge is clearly observed, as well as a $\mathrm{P} \delta$-doped layer with an ultra-thin Si insertion. Additionally, we can actually see the inserted CoFe layer between $\mathrm{Co}_{2} \mathrm{FeAl}_{0.5} \mathrm{Si}_{0.5}$ and Ge.

We first measure current density $(|J|)$-voltage $(V)$ characteristics of the high-quality FM/Ge contacts by using three-terminal voltage measurements, which reveal the FM/Ge junction characteristics without the channel, presented in Figure $2 b$. As mentioned before, the definition of the size of contacts, $S(=L \times W)$, was also depicted in Figure $2 \mathrm{~b}$. Figure $2 \mathrm{c}$, d show $|J|-V$ curves of the used spin-injector and spin-detector contacts with various $S$ at $30 \mathrm{~K}$, respectively.

As previously shown in Ref. [39], we can also see no rectification in the $|J|-V$ curves, indicating the demonstration of the tunneling conduction of electrons through the FM/Ge interfaces. It should be noted that $|J|-V$ characteristics for both spin injector and detector contacts are almost the same. This means that the electrical properties of the FM/Ge Schottky-tunnel contacts are uniform irrespective of $S$. From these results, we can precisely adjust the value of $R A$ to be $\sim 0.4 \mathrm{k} \Omega \cdot \mu \mathrm{m}^{2}$ for all the LSVs used here.

By applying in-plane magnetic fields $\left(B_{\mathrm{y}}\right)$ or out-of-plane magnetic fields $\left(B_{\mathrm{z}}\right)$ to the $\mathrm{FM} / \mathrm{Ge}$ contacts in an LSV, representative four-terminal nonlocal magnetoresistance $\left(\Delta R_{\mathrm{NL}}=\Delta V_{34} / I_{21}\right.$, in Figure 1) data and nonlocal Hanle-effect curves were recorded at $I=-1 \mathrm{~mA}$ at $30 \mathrm{~K}$, as shown in Figure $3 \mathrm{a}, \mathrm{b}$, respectively. We can clearly see hysteretic behavior of the $\Delta R_{\mathrm{NL}}$ depending on the parallel or anti-parallel magnetization configuration between the spin injector and the spin detector in Figure $3 a$. In Figure $3 b$, spin precessional behavior in both parallel and antiparallel magnetic configurations can also be observed. From these data, we can recognize that the generation, manipulation, and detection of pure spin currents through $n$-Ge are reliably demonstrated by all electrical means. By using these reliable devices and techniques, we can explore the influence of the spin absorption effect at the FM/Ge interface.
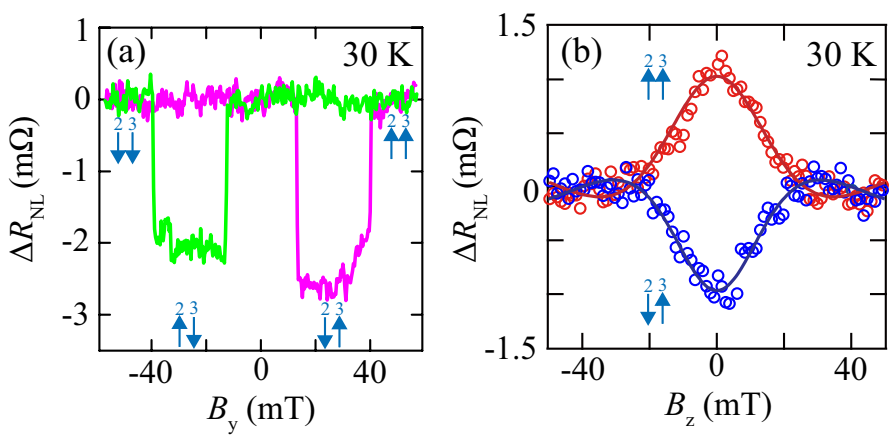

Figure 3. Four-terminal nonlocal (a) magnetoresistance curve and (b) Hanle-effect curves for the parallel and antiparallel magnetization configurations at $30 \mathrm{~K}$. The solid curves in (b) indicate the results fitted to Equation (2) in Ref. [37].

For various LSVs with different $S$, we measured $\Delta R_{\mathrm{NL}}-B_{\mathrm{y}}$ curves at $30 \mathrm{~K}$. Figure 4a shows the plot of the magnitude of $\Delta R_{N L},\left|\Delta R_{N L}\right|$, versus $S$. The inset shows the observed nonlocal spin-valve signals. With increasing $S,\left|\Delta R_{N L}\right|$ markedly decreases, although all the LSVs are fabricated from the same epitaxial layers including FM/Ge. Thus, we can neglect the influences of the difference in the spin diffusion length of the $n$-Ge channel and in the quality of the FM/Ge contacts. As shown in the inset, the shape of the $\Delta R_{\mathrm{NL}}-B_{\mathrm{y}}$ curve becomes unclear for the LSV with the largest $S$. This means that the influence of $S$ on the spin transport in $n$-Ge is more significant than other factors. As mentioned in Section 1, when the spin injection from FM into NM is considered, the spin resistance at the FM contact can be regarded as $2 \lambda \rho /\left[S\left(1-\alpha^{2}\right)\right][30,32]$. If $S$ is changed from $10 \mu \mathrm{m}^{2}$ to $0.8 \mu \mathrm{m}^{2}$, the spin resistance of the FM contact can be varied by more than by a factor of 10 . For our electrical spin injection techniques through FM/Ge Schottky-tunnel contacts, the difference between the $R A$ value of FM/Ge Schottky-tunnel contacts and the spin resistance of Ge is approximately one order of magnitude. Although this condition is satisfied with a condition of the spin injection from FM into SC via tunnel 
barriers [31,49], we should consider the influence of the spin absorption at the FM/SC interface via tunnel barriers, as previously discussed [35].

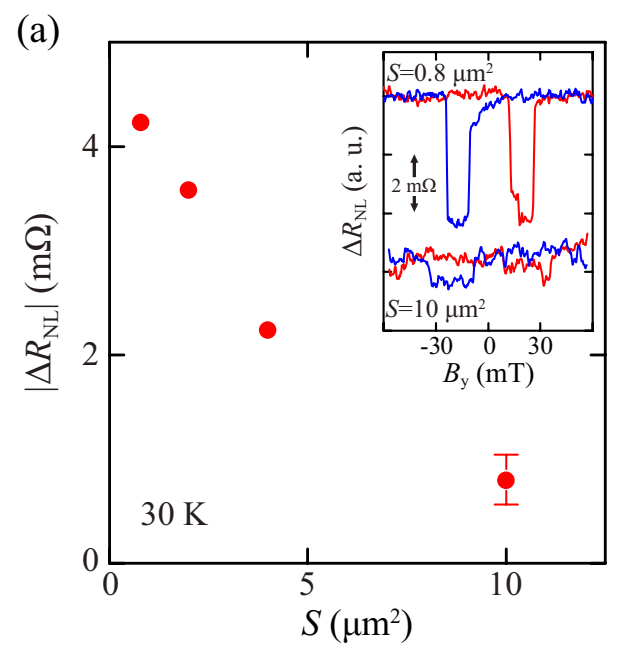

(b)
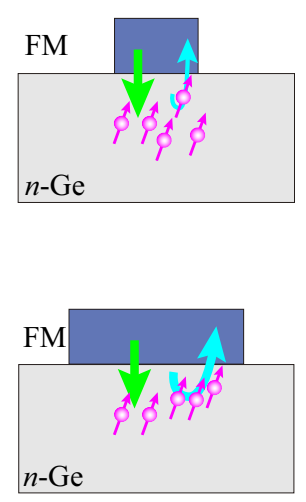

Figure 4. (a) $S$ dependence of $\left|\Delta R_{\mathrm{NL}}\right|$ at $30 \mathrm{~K}$. The inset shows the nonlocal spin signal for the LSVs with $S=0.8$ and $10 \mu \mathrm{m}^{2}$; (b) Schematic diagram of spin absorption effect under the small and large FM contacts.

Figure 5 shows the temperature dependence of $\left|\Delta R_{N L}\right|$ for LSVs with $S=4$ and $10 \mu \mathrm{m}^{2}$. The influence of $S$ becomes remarkable when observing the spin signal at higher temperatures. Despite the same spin relaxation mechanism in the used $n$-Ge channel, the spin signals for $S=10 \mu \mathrm{m}^{2}$ disappear below $100 \mathrm{~K}$, while we can see those for $S=4 \mu \mathrm{m}^{2}$ above $100 \mathrm{~K}$. The same behavior was observed in the spin accumulation in $n$-Si detected by the three terminal method [35]. The features in Figure 5 can also be interpreted by the difference in the spin absorption at the spin injector in the FM/Ge Schottky-tunnel contacts.

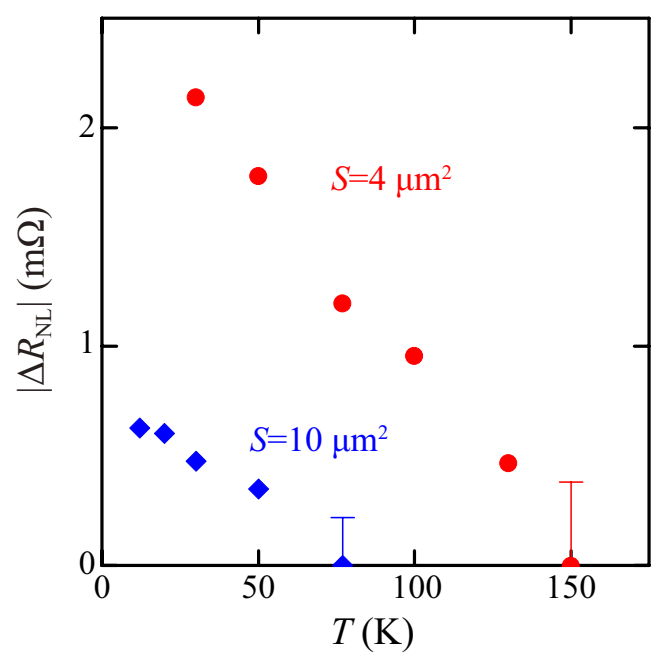

Figure 5. Temperature dependence of spin signal for LSVs with $S=4$ (circle) and 10 (diamond) $\mu \mathrm{m}^{2}$.

Finally, we comment on the importance of geometrical factors of FM contacts in SC spintronic applications. In general, the low $R A$ contacts are first required to reduce the parasitic resistance, leading to the low power consumption. In addition, according to a conventional spin drift-diffusion model [31,49], the ratio of $R A\left(=\mathrm{r}_{b}\right)$ to $\mathrm{r}_{\mathrm{SC}}\left(=\rho_{\mathrm{SC}} \times \lambda_{\mathrm{SC}}\right)$ is important to obtain a large magnetoresistance (MR) ratio, where $\rho_{\mathrm{SC}}$ and $\lambda_{\mathrm{SC}}$ are the resistivity and spin diffusion length of the semiconductor channel, 
respectively. In this study, because $\mathrm{r}_{\mathrm{SC}}$ can be estimated to be $\sim 0.01 \mathrm{k} \Omega \cdot \mu \mathrm{m}^{2}$ at $30 \mathrm{~K}, R A / \mathrm{r}_{\mathrm{SC}}$ is $\sim 40$. For reaching an optimal condition $\left(R A / \mathrm{r}_{\mathrm{SC}} \sim 1\right)$, we should further reduce the value of $R A$. If the value of $R A$ was reduced down to $0.01 \mathrm{k} \Omega \cdot \mu \mathrm{m}^{2}$, the spin absorption could be made even more significant, as mentioned above. Thus, the decrease in $S$ is more effective for SC spintronic applications to achieve highly efficient spin injection from FM into SC without increasing the value of $R A$.

\section{Conclusions}

We studied the influence of the junction size in ferromagnet (FM)/semiconductor (SC) contacts on four-terminal nonlocal spin signals in SC-based lateral spin-valve structures. When we used FM/Ge Schottky-tunnel junctions with relatively low $R A$, the magnitude of the nonlocal spin signal depended clearly on the junction size, meaning the presence of the spin absorption effect at the spin-injector contact. The temperature-dependent spin signal can also be affected by the spin absorption effect. For SC spintronic applications with a low parasitic resistance, we should consider the influence of the spin absorption on the spin-transport signals in SC-based device structures.

Acknowledgments: This work was partially supported by Grants-in-Aid for Scientific Research (A) (No. 16H02333), (S) (No. 17H06120), and Activity Start-up (No. 17H06832) from the Japan Society for the Promotion of Science (JSPS) and a Grant-in-Aid for Scientific Research on Innovative Areas "Nano Spin Conversion Science” (No. 26103003) from the Ministry of Education, Culture, Sports, Science and Technology (MEXT). M.Y. acknowledges scholarships from the Toyota Physical and Chemical Research Institute Foundation. Y.F. acknowledges JSPS Research Fellowships for Young Scientists.

Author Contributions: K.H. and K.S. conceived and designed the experiments; M.Y. and Y.F. performed the experiments; M.Y., Y.F., S.Y. and K.H. analyzed the data; M.Y. and K.H. wrote the paper.

Conflicts of Interest: The authors declare no conflict of interest.

\section{References}

1. Žutić, I.; Fabian, J.; Sarma, S.D. Spintronics: Fundamentals and applications. Rev. Mod. Phys. 2004, 76, 323.

2. Yuasa, S.; Djayaprawira, D.D. Giant tunnel magnetoresistance in magnetic tunnel junctions with a crystalline MgO(0 0 1) barrier. J. Phys. D Appl. Phys. 2007, 40, R337.

3. Tanaka, M.; Sugahara, S. MOS-Based Spin Devices for Reconfigurable Logic. IEEE Trans. Electron. Dev. 2007, 54, 961-976.

4. Taniyama, T.; Wada, E.; Itoh, M.; Yamaguchi, M. Electrical and optical spin injection in ferromagnet/ semiconductor heterostructures. NPG Asia Mater. 2011, 3, 65-73.

5. Hirohata, A.; Takanashi, K. Future perspectives for spintronic devices. J. Phys. D Appl. Phys. 2014, 47, 193001.

6. Lou, X.; Adelmann, C.; Crooker, S.A.; Garlid, E.S.; Zhang, J.; Reddy, K.S.M.; Flexner, S.D.; Palmstrøm, C.J.; Crowell, P.A. Electrical detection of spin transport in lateral ferromagnet-semiconductor devices. Nat. Phys. 2007, 3, 197-202.

7. Salis, G.; Fuhrer, A.; Schlittler, R.R.; Gross, L.; Alvarado, S.F. Temperature dependence of the nonlocal voltage in an Fe/GaAs electrical spin-injection device. Phys. Rev. B 2010, 81, 205323.

8. Song, C.; Sperl, M.; Utz, M.; Ciorga, M.; Woltersdorf, G.; Schuh, D.; Bougeard, D.; Back, C.H.; Weiss, D. Proximity Induced Enhancement of the Curie Temperature in Hybrid Spin Injection Devices. Phys. Rev. Lett. 2011, 107, 056601.

9. Bruski, P.; Manzke, Y.; Farshchi, R.; Brandt, O.; Herfort, J.; Remsteiner, M. All-electrical spin injection and detection in the $\mathrm{Co}_{2} \mathrm{FeSi} / \mathrm{GaAs}$ hybrid system in the local and non-local configuration. Appl. Phys. Lett. 2013, 103, 052406.

10. Appelbaum, I.; Huang, B.; Monsma, D.J. Electronic measurement and control of spin transport in silicon. Nature 2007, 447, 295-298.

11. Van't Erve, O.M.J.; Hanbicki, A.T.; Holub, M.; Awo-Affouda, C.; Li, C.H.; Thompson, P.E.; Jonker, B.T. Electrical injection and detection of spin-polarized carriers in silicon in a lateral transport geometry. Appl. Phys. Lett. 2007, 91, 212109.

12. Dash, S.P.; Sharma, S.; Patel, R.S.; Jong, M.P.; Jansen, R. Electrical creation of spin polarization in silicon at room temperature. Nature 2009, 462, 491-494. 
13. Ando, Y.; Hamaya, K.; Kasahara, K.; Kishi, Y.; Ueda, K.; Sawano, K.; Sadoh, T.; Miyao, M. Electrical injection and detection of spin-polarized electrons in silicon through an $\mathrm{Fe}_{3} \mathrm{Si} / \mathrm{Si}$ Schottky tunnel barrier. Appl. Phys. Lett. 2009, 94, 182105.

14. Ando, Y.; Maeda, Y.; Kasahara, K.; Yamada, S.; Masaki, K.; Hoshi, Y.; Sawano, K.; Izunome, K.; Sakai, A.; Miyao, M.; et al. Electric-field control of spin accumulation signals in silicon at room temperature. Appl. Phys. Lett. 2011, 99, 132511.

15. Sasaki, T.; Oikawa, T.; Suzuki, T.; Shiraishi, M.; Suzuki, Y.; Tagami, K. Electrical Spin Injection into Silicon Using MgO Tunnel Barrier. Appl. Phys. Exp. 2009, 2, 053003.

16. Suzuki, T.; Sasaki, T.; Oikawa, T.; Shiraishi, M.; Suzuki, Y.; Noguchi, K. Room-Temperature Electron Spin Transport in a Highly Doped Si Channel. Appl. Phys. Exp. 2011, 4, 023003.

17. Saito, Y.; Tanamoto, T.; Ishikawa, M.; Sugiyama, H.; Inokuchi, T.; Hamaya, K.; Tezuka, N. Local magnetoresistance through $\mathrm{Si}$ and its bias voltage dependence in ferromagnet/ $\mathrm{MgO} /$ silicon-on-insulator lateral spin valves. J. Appl. Phys. 2014, 115, 17C514.

18. Ishikawa, M.; Oka, T.; Fujita, Y.; Sugiyama, H.; Saito, Y.; Hamaya, K. Spin relaxation through lateral spin transport in heavily doped n-type silicon. Phys. Rev. B 2017, 95, 115302.

19. Zhou, Y.; Han, W.; Chang, L.-T.; Xiu, F.; Wang, M.; Oehme, M.; Fischer, I.A.; Schulze, J.; Kawakami, R.K.; Wang, K.L. Electrical spin injection and transport in germanium. Phys. Rev. B 2011, 84, 125323.

20. Saito, H.; Watanabe, S.; Mineno, Y.; Sharma, S.; Jansen, R.; Yuasa, S.; Ando, K. Electrical creation of spin accumulation in p-type germanium. Sol. State Commun. 2011, 151, 1159-1161.

21. Jeon, K.-R.; Min, B.-C.; Jo, Y.-H.; Lee, H.-S.; Shin, I.-J.; Park, C.-Y.; Park, S.-Y.; Shin, S.-C. Electrical spin injection and accumulation in CoFe/MgO/Ge contacts at room temperature. Phys. Rev. B 2011, 84, 165315.

22. Kasahara, K.; Baba, Y.; Yamane, K.; Ando, Y.; Yamada, S.; Hoshi, Y.; Sawano, K.; Miyao, M.; Hamaya, K. Spin accumulation created electrically in an n-type germanium channel using Schottky tunnel contacts. J. Appl. Phys. 2012, 111, 07C503.

23. Jain, A.; Rojas-Sanchez, J.-C.; Cubukcu, M.; Peiro, J.; Le Breton, J.C.; Prestat, E.; Vergnaud, C.; Louahadj, L.; Portemont, C.; Ducruet, C.; et al. Crossover from Spin Accumulation into Interface States to Spin Injection in the Germanium Conduction Band. Phys. Rev. Lett. 2012, 109, 106603.

24. Kawano, M.; Santo, K.; Ikawa, M.; Yamada, S.; Kanashima, T.; Hamaya, K. Spin transport in $p$-Ge through a vertically stacked $\mathrm{Ge} / \mathrm{Fe}_{3} \mathrm{Si}$ junction. Appl. Phys. Lett. 2016, 109, 022406.

25. Fujita, Y.; Yamada, M.; Tsukahara, M.; Oka, T.; Yamada, S.; Kanashima, T.; Sawano, K.; Hamaya, K. Spin Transport and Relaxation up to $250 \mathrm{~K}$ in Heavily Doped n-Type Ge Detected Using $\mathrm{Co}_{2} \mathrm{FeAl}_{0.5} \mathrm{Si}_{0.5}$ Electrodes. Phys. Rev. Appl. 2017, 8, 014007.

26. Yamada, M.; Tsukahara, M.; Fujita, Y.; Naito, T.; Yamada, S.; Sawano, K.; Hamaya, K. Room-temperature spin transport in $n$-Ge probed by four-terminal nonlocal measurements. Appl. Phys. Express 2017, 10, 093001.

27. Dushenko, S.; Koike, M.; Ando, Y.; Shinjo, T.; Myronov, M.; Shiraishi, M. Experimental Demonstration of Room-Temperature Spin Transport in n-Type Germanium Epilayers. Phys. Rev. Lett. 2015, 114, 196602.

28. Schmidt, G.; Ferrand, D.; Molemkamp, L.W.; Filip, A.T.; Van Wees, B.J. Fundamental obstacle for electrical spin injection from a ferromagnetic metal into a diffusive semiconductor. Phys. Rev. B 2000, 62, R4790.

29. Rashba, E.I. Theory of electrical spin injection: Tunnel contacts as a solution of the conductivity mismatch problem. Phys. Rev. B 2000, 62, R16267.

30. Takahashi, S.; Maekawa, S. Spin injection and detection in magnetic nanostructures. Phys. Rev. B 2003, $67,052409$.

31. Fert, A.; Jaffrès, H. Conditions for efficient spin injection from a ferromagnetic metal into a semiconductor. Phys. Rev. B 2001, 64, 184420.

32. Kimura, T.; Otani, Y.; Hamrle, J. Enhancement of spin accumulation in a nonmagnetic layer by reducing junction size. Phys. Rev. B 2006, 73, 132405.

33. Yang, T.; Kimura, T.; Otani, Y. Giant spin-accumulation signal and pure spin-current-induced reversible magnetization switching. Nat. Phys. 2008, 4, 851-854.

34. Nomura, T.; Ohnishi, K.; Kimura, T. Geometrical dependence of spin current absorption into a ferromagnetic nanodot. J. Appl. Phys. 2016, 120, 142121.

35. Ishikawa, M.; Sugiyama, H.; Inokuchi, T.; Hamaya, K.; Saito, Y. Effect of the interface resistance of CoFe/MgO contacts on spin accumulation in silicon. Appl. Phys. Lett. 2012, 100, 252404. 
36. Johnson, M.; Silsbee, R.H. Interfacial Charge-Spin Coupling: Injection and Detection of Spin Magnetization in Metals. Phys. Rev. Lett. 1985, 55, 1790-1793.

37. Jedema, F.J.; Heersche, H.B.; Filip, A.T.; Baselmans, J.J.A.; van Wees, B.J. Electrical detection of spin precession in a metallic mesoscopic spin valve. Nature 2002, 416, 713-716.

38. Kimura, T.; Otani, Y. Spin transport in lateral ferromagnetic/nonmagnetic hybrid structures. J. Phys. Condens. Matter 2007, 19, 165216.

39. Fujita, Y.; Yamada, M.; Yamada, S.; Kanashima, T.; Sawano, K.; Hamaya, K. Temperature-independent spin relaxation in heavily doped $n$-type germanium. Phys. Rev. B 2016, 94, 245302.

40. Yamada, M.; Fujita, Y.; Tsukahara, M.; Yamada, S.; Sawano, K.; Hamaya, K. Large impact of impurity concentration on spin transport in degenerate $n$-Ge. Phys. Rev. B 2017, 95, 161304(R).

41. Cesari, S.D.; Vitiello, E.; Giorgioni, A.; Pezzoli, F. Progress towards Spin-Based Light Emission in Group IV Semiconductors. Electronics 2017, 6, 19.

42. Sawano, K.; Hoshi, Y.; Kubo, S.; Arimoto, K.; Yamanaka, J.; Nakagawa, K.; Hamaya, K.; Miyao, M.; Shiraki, Y. Structural and electrical properties of Ge(111) films grown on Si(111) substrates and application to Ge(111)-on-Insulator. Thin Solid Films 2016, 613, 24-28.

43. Yamada, M.; Sawano, K.; Uematsu, M.; Itoh, K.M. Suppression of surface segregation of the phosphorous $\delta$-doping layer by insertion of an ultra-thin silicon layer for ultra-shallow Ohmic contacts on n-type germanium. Appl. Phys. Lett. 2015, 107, 132101.

44. Yamada, M.; Fujita, Y.; Yamada, S.; Kanashima, T.; Sawano, K.; Hamaya, K. Control of electrical properties in Heusler-alloy/Ge Schottky tunnel contacts by using phosphorous $\delta$-doping with Si-layer insertion. Mater. Sci. Semicond. Process. 2017, 70, 83-85.

45. Shan, R.; Sukegawa, H.; Wang, W.H.; Kodzuka, M.; Furubayashi, T.; Ohkubo, T.; Mitani, S.; Inomata, K.; Hono, K. Demonstration of Half-Metallicity in Fermi-Level-Tuned Heusler Alloy $\mathrm{Co}_{2} \mathrm{FeAl}_{0.5} \mathrm{Si}_{0.5}$ at Room Temperature. Phys. Rev. Lett. 2009, 102, 246601.

46. Hamaya, K.; Itoh, H.; Nakatsuka, O.; Ueda, K.; Yamamoto, K.; Itakura, M.; Taniyama, T.; Ono, T.; Miyao, M. Ferromagnetism and Electronic Structures of Nonstoichiometric Heusler-Alloy $\mathrm{Fe}_{3-x} \mathrm{Mn}_{x} \mathrm{Si}$ Epilayers Grown on Ge(111). Phys. Rev. Lett. 2009, 102, 137204.

47. Yamada, S.; Sagar, J.; Honda, S.; Lari, L.; Takemoto, G.; Itoh, H.; Hirohata, A.; Mibu, K.; Miyao, M.; Hamaya, K. Room-temperature structural ordering of a Heusler compound $\mathrm{Fe}_{3} \mathrm{Si}$. Phys. Rev. B 2012, 86, 174406.

48. Ikawa, M.; Kawano, M.; Sakai, S.; Yamada, S.; Kanashima, T.; Hamaya, K. Influence of the Ge diffusion on the magnetic and structural properties in $\mathrm{Fe}_{3} \mathrm{Si}$ and $\mathrm{CoFe}$ epilayers grown on Ge. J. Cryst. Growth 2017, $468,676-679$.

49. Fert, A.; George, J.-M.; Jaffrès, H.; Mattana, R. Semiconductors Between Spin-Polarized Sources and Drains. IEEE Trans. Electron. Dev. 2007, 54, 921-932.

(C) 2018 by the authors. Licensee MDPI, Basel, Switzerland. This article is an open access article distributed under the terms and conditions of the Creative Commons Attribution (CC BY) license (http://creativecommons.org/licenses/by/4.0/). 